\title{
Evaluating the effect of magnetocaloric properties on magnetic refrigeration performance
}

\section{Engelbrecht, Kurt; Bahl, Christian Robert Haffenden}

Published in:

Journal of Applied Physics

Link to article, DOI:

$10.1063 / 1.3525647$

Publication date:

2010

Document Version

Publisher's PDF, also known as Version of record

Link back to DTU Orbit

Citation (APA):

Engelbrecht, K., \& Bahl, C. R. H. (2010). Evaluating the effect of magnetocaloric properties on magnetic refrigeration performance. Journal of Applied Physics, 108(12), 123918. https://doi.org/10.1063/1.3525647

\section{General rights}

Copyright and moral rights for the publications made accessible in the public portal are retained by the authors and/or other copyright owners and it is a condition of accessing publications that users recognise and abide by the legal requirements associated with these rights.

- Users may download and print one copy of any publication from the public portal for the purpose of private study or research.

- You may not further distribute the material or use it for any profit-making activity or commercial gain

- You may freely distribute the URL identifying the publication in the public portal

If you believe that this document breaches copyright please contact us providing details, and we will remove access to the work immediately and investigate your claim. 


\title{
Evaluating the effect of magnetocaloric properties on magnetic refrigeration performance
}

\author{
K. Engelbrecht ${ }^{\text {a) }}$ and C. R. H. Bahl \\ Risф National Laboratory for Sustainable Energy, Frederiksborgvej 399, DK-4000 Roskilde, Denmark
}

(Received 19 August 2010; accepted 10 November 2010; published online 30 December 2010)

\begin{abstract}
Active magnetic regenerator (AMR) refrigerators represent an alternative to vapor compression technology that relies on the magnetocaloric effect in a solid refrigerant. Magnetocaloric materials are in development and properties are reported regularly. Recently, there has been an emphasis on developing materials with a high entropy change with magnetization while placing lower emphasis on the adiabatic temperature change. This work uses model magnetocaloric materials and a numerical AMR model to predict how the temperature change and entropy change with magnetization interact and how they affect the performance of a practical system. The distribution of the magnetocaloric effect as a function of temperature was also studied. It was found that the adiabatic temperature change in a magnetocaloric material can be more important than the isothermal entropy change for certain conditions. A material that exhibits a sharp peak in isothermal entropy change was shown to produce a significantly lower cooling power than a material with a wide peak in a practical AMR system. (0) 2010 American Institute of Physics.
\end{abstract}

[doi:10.1063/1.3525647]

\section{INTRODUCTION}

Active magnetic regenerative (AMR) refrigerators are a potentially environmentally friendly alternative to vapor compression technology that may be used for airconditioning, refrigeration, and heat pump applications. Rather than using a gaseous refrigerant, AMRs use magnetocaloric materials (MCMs) that have a coupling between their thermodynamic properties and internal magnetic field. Assuming the material properties are independent of pressure the entropy of a MCM can be expressed as

$$
d s=\frac{c_{B}}{T} d T+\left(\frac{\partial s}{\partial B}\right)_{T} d B,
$$

where $B$ is the magnetic field and $c_{B}$ is the specific heat at constant magnetic field. As the magnetic field is increased the entropy of a MCM decreases as it moves to a more ordered state. Equation (1) illustrates that for a material with a positive magnetocaloric effect, the temperature of the material must increase when the material is magnetized adiabatically to maintain constant entropy. The magnitude of the temperature increase is related to the specific heat and the entropy change with magnetization of the material.

AMRs are a developing technology and there is much research effort currently focused on improving AMR performance. The properties of many new MCMs have been reported recently; ${ }^{1}$ however, it is not certain which properties are most important when evaluating new materials. Several figures of merit for evaluating magnetocaloric properties have been proposed, but these figures ignore important aspects of the AMR cycle. This paper investigates several important magnetocaloric properties and demonstrates their effect on AMR performance.

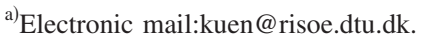

The earliest magnetic refrigerators used a one-shot demagnetization cycle with a temperature span that was limited by the adiabatic temperature change of the MCM. Such a device was used to create a cooling device able to cool below $1 \mathrm{~K}^{2}{ }^{2}$ The operating temperature span of magnetic refrigerators can be dramatically increased by using a regenerative cycle, as demonstrated by Ref. 3. Since 1976, many new devices have been reported, ${ }^{4}$ with modern AMRs generally using permanent magnets with regenerators made of packed spheres, packed particles, or parallel plates of MCM. The AMR cycle uses a heat transfer fluid to transport the heat generated from magnetizing and demagnetizing the MCM to the hot and cold reservoirs. The AMR cycle has four basic processes: magnetization, the cold-to-hot blow, demagnetization, and the hot-to-cold blow. During magnetization, the temperature of the MCM increases, then fluid is pumped from the cold reservoir to the hot reservoir in order to reject the magnetic work to ambient. The regenerator is then demagnetized, causing a decrease in temperature and a cooling load is accepted from the cooled space by pumping fluid from the hot reservoir across the regenerator and into the cold reservoir. The system performance is mostly a function of the MCM, heat transfer characteristics in the regenerator, and cycle parameters such as frequency and fluid flow rate.

Developing and characterizing new MCMs is an active research topic and the properties of many new compositions are reported each year. ${ }^{5}$ The two most cited properties for potential AMR materials are the adiabatic temperature change with magnetization, $\Delta T_{\text {ad }}$, and the isothermal entropy change with magnetization, $\Delta s_{\text {mag. }}$. Much of the recent research has focused on developing first order magnetic transition (FOMT) materials that exhibit high $\Delta s_{\text {mag }}$ but often have lower $\Delta T_{\text {ad }}$ than second order transition materials such as Gd. Some FOMT materials of interest that have recently been reported are $\mathrm{Gd}_{5} \mathrm{Si}_{2} \mathrm{Ge}_{2},{ }^{6} \mathrm{La}(\mathrm{Fe}, \mathrm{Si})_{13},{ }^{7}$ and $\mathrm{MnFe}(\mathrm{P}$ and 

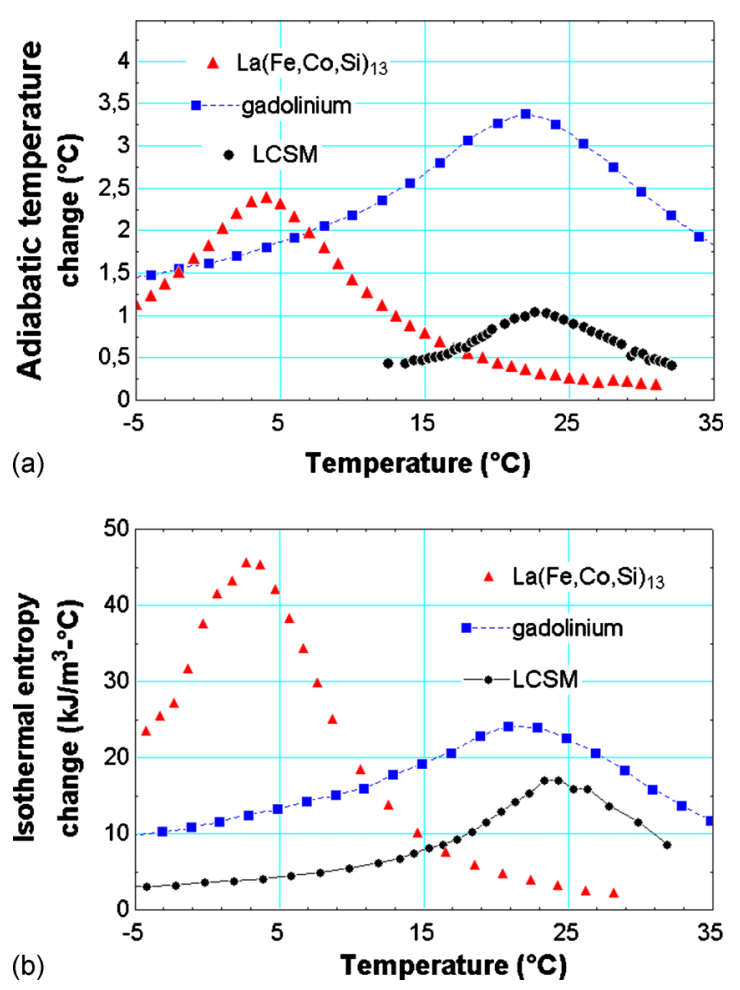

FIG. 1. (Color online) The adiabatic temperature change (a) and volumetric isothermal entropy change (b) of $\mathrm{LaFe}_{11.06} \mathrm{Co}_{0.86} \mathrm{Si}_{1.08}$ gadolinium $(\mathrm{Gd})$, and $\mathrm{La}_{0.67} \mathrm{Ca}_{0.26} \mathrm{Sr}_{0.07} \mathrm{Mn}_{1.05} \mathrm{O}_{3}$ (an LCSM compound) when magnetized from 0 to $1 \mathrm{~T}$ as a function of temperature (Ref. 13).

As). ${ }^{8}$ Many recent publications on MCM properties (such as Ref. 9) report only $\Delta s_{\text {mag }}$ without $\Delta T_{\text {ad }}$ or the specific heat while others present both properties (Refs. 10-12 among others). However, it is not well understood how the combination of the two properties affect practical AMR performance or what ideal MCM properties are. This paper uses a onedimensional (1D) numerical model of a simple AMR system to predict how variations in $\Delta T_{\text {ad }}$ and $\Delta s_{\text {mag }}$ interact and which MCM properties are more desirable for AMR applications.

The magnetocaloric effect for most materials exhibiting a large effect near room temperature is highly temperature dependent. $\Delta s_{\text {mag }}$ and $\Delta T_{\text {ad }}$ as a function of temperature as reported by Ref. 13 for three candidate MCMs are shown in Fig. 1. The figure shows that magnetocaloric properties are generally highly nonlinear and that the magnitude of $\Delta s_{\text {mag }}$ and $\Delta T_{\text {ad }}$ can differ significantly between materials, due to differences in the value of the specific heat for each material. The figure shows that Gd has a relatively high $\Delta T_{\text {ad }}$ but a moderate $\Delta s_{\text {mag }}$. Gd is the most common MCM reported in AMR prototypes to date ${ }^{4}$ and the highest operating temperature spans are generally achieved using regenerators made of Gd and its alloys, such as $\mathrm{Gd}_{1-\mathrm{x}} \mathrm{Er}_{\mathrm{x}}$ or $\mathrm{Gd}_{1-\mathrm{x}} \mathrm{Tb}_{\mathrm{x}}{ }^{14}$

\section{A. Thermal processes in an AMR}

The thermal and hydraulic interactions in an AMR device are the same as those described for passive regenerator applications ${ }^{15}$ with the addition of a magnetic interaction term. Including magnetic interaction terms and considering the equations only in the fluid flow direction, the 1D AMR governing equations are shown below. The governing equations for the fluid phase in an AMR are given in Eq. (2) with subscripts $f$ and $s$ indicating fluid and solid.

$$
\dot{m}_{\mathrm{f}} c_{\mathrm{f}} \frac{\partial T_{\mathrm{f}}}{\partial x}+h A_{\mathrm{HT}}\left(T_{\mathrm{f}}-T_{\mathrm{s}}\right)+\rho_{\mathrm{f}} A_{\mathrm{c}} \epsilon c_{\mathrm{f}} \frac{\partial T_{\mathrm{f}}}{\partial t}-k_{\mathrm{disp}} A_{\mathrm{c}} \frac{\partial^{2} T_{\mathrm{f}}}{\partial x^{2}}=0,
$$

where $T$ is temperature, $\varrho$ is density, $c$ is specific heat, $h$ is the heat transfer coefficient, $A_{\mathrm{HT}}$ is the area for heat transfer, $\epsilon$ is the porosity, $\dot{m}_{\mathrm{f}}$ is the fluid mass flow rate, and $A_{\mathrm{C}}$ is the cross sectional area. The terms represent (in order from left to right) the enthalpy change in the flow, heat transfer from the fluid to the solid, energy storage, and energy transfer due to axial dispersion associated with mixing of the fluid. Viscous dissipation due to pumping losses is ignored. The governing equation for the solid regenerator material is

$$
\begin{aligned}
& h A_{\mathrm{HT}}\left(T_{\mathrm{f}}-T_{\mathrm{s}}\right)+k_{\mathrm{eff}} \frac{\partial^{2} T_{\mathrm{s}}}{\partial x^{2}} \\
& =(1-\epsilon) \rho_{\mathrm{s}} T_{\mathrm{s}}\left(\frac{\partial s_{\mathrm{s}}}{\partial B}\right) \frac{\partial B}{\mathrm{~T}}+(1-\epsilon) \rho_{\mathrm{s}} c_{\mathrm{B}, \mathrm{s}} \frac{\partial T_{\mathrm{s}}}{\partial t},
\end{aligned}
$$

where $B$ is the internal magnetic field of the solid regenerator and $k_{\text {eff }}$ is the effective thermal conductivity of the fluid and solid matrix. The terms represent heat transfer from the fluid to the regenerator, nondispersive, or static axial conduction (through the composite of the regenerator and fluid), magnetic work transfer, and energy storage.

Examination of Eqs. (2) and (3) reveals that the most important physical interactions in an AMR device are the magnetocaloric effect in the solid material, heat transfer between the fluid and solid, energy storage in the solid and fluid phases, and axial conduction through the regenerator from the hot reservoir to the cold reservoir. Pumping losses are ignored in this study because they are dependent on regenerator geometry and operating conditions and not directly to magnetocaloric properties. The effect of varying axial conduction losses is not considered to reduce the parameter space. This work considers how MCM selection affects AMR performance through heat transfer from the solid to the fluid and the magnetic work into the system. The magnetic work term is straightforward, as it is directly proportional to $\Delta s_{\text {mag }}$ of the material and the magnetic field change, but $\Delta T_{\text {ad }}$ also plays an important role in increasing the temperature difference between the solid and fluid and facilitates heat transfer between the fluid and solid across a temperature span and heat rejection to a warmer thermal reservoir. Thermal storage in the solid is also important because the specific heat of MCMs can vary widely. ${ }^{1}$

\section{B. Current methods to evaluate MCMs}

As new MCMs are fabricated and characterized, it is necessary to compare them to the current state of the art materials. There are several methods currently used to estimate the potential performance of a MCM used in a magnetic refrigerator. The first is to simply integrate the isothermal entropy change with magnetization over the working 
temperature span of the regenerator ${ }^{1}[$ Eq. (4)]. This is referred to as the refrigeration capacity of the material

$$
R_{\mathrm{CAP}}=\int_{T_{C}}^{T_{H}} \Delta s_{\mathrm{mag}}(T) d T
$$

Another method is to calculate the relative cooling power, $R C P$, of the material. ${ }^{16} R C P$ is defined as the product of the peak value of $\Delta s_{\text {mag }}$ and the temperature range at half maximum, $\Delta T_{\mathrm{THM}}$, which is defined as the temperature span between the two points where the entropy change with magnetization is half the peak value

$$
R C P=\Delta s_{\text {mag, } \mathrm{MAX}} \Delta T_{\mathrm{THM}} .
$$

In the regenerative cycle, there is a temperature gradient from the cold reservoir to the hot reservoir in the direction of flow after steady state has been achieved. The temperature gradient in the solid material means that each location along the flow direction of the regenerator is potentially at a different temperature, and, therefore, undergoes a unique thermodynamic cycle. Neither of the techniques described above accounts for the different cycle experienced by each position in the regenerator and at times do not adequately describe the magnetocaloric properties of a material used in an AMR. The figures of merit from Eqs. (4) and (5) ignore the heat transfer process between the solid and fluid in an AMR and can, therefore, be misleading when evaluating an MCM. The refrigeration capacity, $R_{\mathrm{CAP}}$ is positive for any given temperature span, even if $\Delta T_{\mathrm{ad}}$ is zero for a large portion of the operating temperature. The $R C P$ of a material is independent of operating temperatures and is only applicable when the material operates near its Curie temperature. The remainder of this work studies how both $\Delta T_{\text {ad }}$ and $\Delta s_{\text {mag }}$ affect the performance of a practical AMR.

\section{MCM PROPERTIES}

Because the purpose of this work is to study the effects of varying properties on AMR performance, it is most convenient to use modeled materials. Examining Eqs. (2) and (3), the solid material properties that are relevant for an AMR are density, thermal conductivity, specific heat at constant magnetic field, $\Delta T_{\text {ad }}$, and $\Delta s_{\text {mag }}$. The density and thermal conductivity are both held constant for all materials in this work. The specific heat, $\Delta T_{\text {ad }}$ and $\Delta s_{\text {mag }}$ are coupled through the entropy curves, and if two are specified, the third can be calculated. Assuming the MCM properties are independent of pressure, the zero-field entropy of the material can be calculated by integrating the assumed zero-field specific heat

$$
s(T, B=0)=\int_{0}^{T}\left(\frac{c_{\mathrm{B}, \mathrm{s}}}{T} d T\right) .
$$

The entropy for each magnetic field is then calculated by either shifting the zero-field entropy along the temperature axis by $\Delta T_{\text {ad }}$ or shifting it along the entropy axis by $\Delta s_{\text {mag. }}$. The specific heat at nonzero magnetic fields must be calculated from the entropy curve at the desired field. Three sets of MCMs were studied: a set with constant $R_{\mathrm{CAP}}$, constant $\Delta s_{\text {mag }}$ and varying $\Delta T_{\text {ad }}$, a set with constant $\Delta T_{\text {ad }}$ and varying
TABLE I. Magnetocaloric characteristics of a series of materials with constant adiabatic temperature change and varying specific heat.

\begin{tabular}{ccccr}
\hline \hline Material & $\begin{array}{c}\text { Specific heat } \\
(\mathrm{J} / \mathrm{kg} \mathrm{K})\end{array}$ & $\begin{array}{c}\Delta T_{\text {ad }} \\
(\mathrm{K})\end{array}$ & $\begin{array}{c}\Delta s_{\text {mag }}(\mathrm{avg}) \\
(\mathrm{J} / \mathrm{kg} \mathrm{K})\end{array}$ & $\begin{array}{r}R_{\mathrm{CAP}} \\
(\mathrm{J} / \mathrm{kg})\end{array}$ \\
\hline 1 & 250 & 4 & -3.5 & 51.6 \\
2 & 500 & 4 & -7.0 & 103.3 \\
3 & 1000 & 4 & -14.0 & 206.6 \\
4 & 1500 & 4 & -21.1 & 309.9 \\
\hline \hline
\end{tabular}

$\Delta s_{\text {mag }}$ and thus varying $R_{\mathrm{CAP}}$, and a set with Lorentzian distributions of $\Delta s_{\text {mag }}$ and constant $\Delta T_{\text {ad }}$ and constant $R_{\mathrm{CAP}}$. The sets of modeled properties can be used to study the effect on AMR performance of varying $\Delta T_{\mathrm{ad}}, \Delta s_{\mathrm{mag}}$, and the shape of the $\Delta s_{\text {mag }}$ curve, respectively, while the other pertinent material properties are held constant. The properties of the materials with intermediate values were chosen to be similar to $\mathrm{Gd}$ or a composition of $\mathrm{La}(\mathrm{Fe}, \mathrm{Co}, \mathrm{Si})_{13}$ but the extreme properties are strictly theoretical. For example, no material with a $\Delta T_{\text {ad }}$ of $13.8 \mathrm{~K}$ at $2 \mathrm{~T}$ is currently known, but it is of interest to model.

The magnetocaloric properties of the first two sets of materials that are used for AMR system simulations are summarized in Tables I and II. $R_{\mathrm{CAP}}$ was calculated for each material assuming the AMR operates between 285 and 300 $\mathrm{K}$. The values of $\Delta T_{\mathrm{ad}}$ and $\Delta s_{\mathrm{mag}}$ were chosen to be on the same order of magnitude as $\mathrm{Gd}$ (Ref. 17) or $\mathrm{La}(\mathrm{Fe}, \mathrm{Si})_{13}$ (Ref. 18) for a maximum magnetic field of $2 \mathrm{~T}$. The thermal conductivity for each material was set to a temperatureindependent value of $10 \mathrm{~W} / \mathrm{m} \mathrm{K}$ based on property measurements by. ${ }^{19}$

Tables I and II illustrate the relationship between specific heat, $\Delta T_{\text {ad }}$, and $\Delta s_{\text {mag. }}$. For a given $\Delta s_{\text {mag }}, \Delta T_{\text {ad }}$ decreases as the specific heat increases while $R_{\mathrm{CAP}}$ remains constant.

The final material set was developed to study how the shape of the magnetocaloric effect as a function of temperature affects AMR performance. For this study, the shape of the $\Delta s_{\text {mag }}$ curve illustrated in Fig. 1 is approximated as a Lorentzian curve with an assumed Curie temperature. To fully specify the magnetocaloric properties, $\Delta T_{\text {ad }}$ is assumed to be a constant value of $4 \mathrm{~K}$ and $R_{\mathrm{CAP}}$ is set to $103 \mathrm{~J} / \mathrm{kg}$, corresponding to material 2 from Table I. The formula used to calculate the zero-field heat capacity is given in Eq. (7)

$$
c_{\mathrm{B}=0, \mathrm{~s}}=\frac{c_{\text {peak }}}{\pi} \frac{\Gamma}{\left(T-T_{\text {Curie }}\right)^{2}+\Gamma^{2}},
$$

where $\Gamma$ is a parameter that determines the width of the specific heat curve. Because $\Delta T_{\text {ad }}$ is assumed constant for

TABLE II. Magnetocaloric characteristics of a series of materials with constant entropy change with magnetization and varying specific heat.

\begin{tabular}{ccccc}
\hline \hline Material & $\begin{array}{c}\text { Specific heat } \\
(\mathrm{J} / \mathrm{kg} \mathrm{K})\end{array}$ & $\begin{array}{c}\Delta T_{\text {ad }}(\mathrm{avg}) \\
(\mathrm{K})\end{array}$ & $\begin{array}{c}\Delta s_{\text {mag }} \\
(\mathrm{J} / \mathrm{kg} \mathrm{K})\end{array}$ & $\begin{array}{c}R_{\mathrm{CAP}} \\
(\mathrm{J} / \mathrm{kg})\end{array}$ \\
\hline \multirow{2}{*}{1} & 125 & 13.8 & -6 & 90 \\
2 & 250 & 6.8 & -6 & 90 \\
3 & 500 & 3.4 & -6 & 90 \\
4 & 1000 & 1.7 & -6 & 90 \\
\hline \hline
\end{tabular}




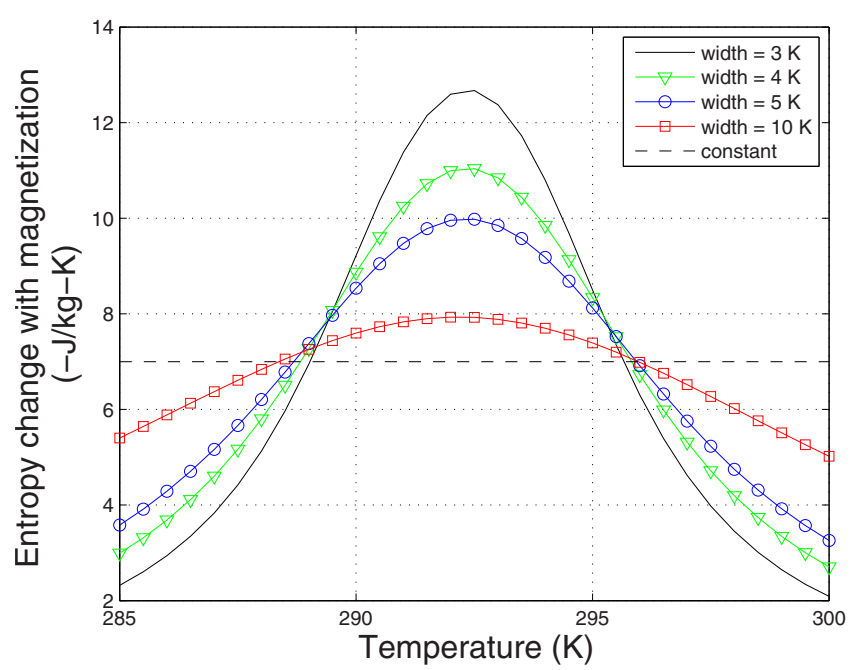

FIG. 2. (Color online) The entropy change with magnetization as a function of temperature for several materials listed in Table III.

these materials, the zero-field specific heat calculated in Eq. (7) determines the $\Delta s_{\text {mag }}$ curve. The term $c_{\text {peak }}$ is determined iteratively for each value of $\Gamma$ by varying the value until the desired value of $R_{\mathrm{CAP}}$ is achieved.

Material properties were modeled for $\Gamma$ values of 3, 4, 5, and 10. Material 2 from Table I can be considered part of the final set with a $\Gamma$ that approaches infinity. The Curie temperature, as defined by the temperature where $\Delta s_{\operatorname{mag}}$ is maximum, of all materials in the final set is set to $292.5 \mathrm{~K}$, which is the average of the hot and cold reservoir temperatures. A plot of the calculated $\Delta s_{\text {mag }}$ curves for the materials from this final material set is shown in Fig. 2 and a summary of the materials is given in Table III.

\section{NUMERICAL MODELING TECHNIQUE}

The nonlinear nature of magnetocaloric properties makes numerical modeling of an AMR system necessary. Several detailed AMR models have been presented recently, including 1D porous models (e.g., Refs. 20-23), two-dimensional (2D) models of parallel plate regenerators, ${ }^{24,25}$ a $2 \mathrm{D}$ porous model, ${ }^{26}$ and a full three-dimensional (3D) model. ${ }^{27}$ Porous models require correlations to calculate heat transfer and pressure drop inside the regenerator bed, while the $2 \mathrm{D}$ plate models and the 3D model solve the coupled fluid flow and heat transfer equations directly. Beside the geometry modeled, the different models often use different approaches to implement the magnetocaloric effect in the solid material. Many models treat the magnetocaloric effect as a heat source

TABLE III. Magnetocaloric characteristics of a series of materials with constant refrigeration capacity and varying standard deviation of entropy change with magnetization.

\begin{tabular}{ccccc}
\hline \hline & $\begin{array}{c}\text { Std. dev. } \\
\text { Material }\end{array}$ & $\begin{array}{c}\Delta T_{\text {ad }} \\
(\mathrm{K})\end{array}$ & $\begin{array}{c}\text { Max. } \Delta s_{\text {mag }} \\
(\mathrm{J} / \mathrm{kg} \mathrm{K})\end{array}$ & $\begin{array}{c}R_{\mathrm{CAP}} \\
(\mathrm{J} / \mathrm{kg})\end{array}$ \\
\hline 1 & 3 & 4 & -10.0 & 103 \\
2 & 4 & 4 & -10.1 & 103 \\
3 & 5 & 4 & -9.4 & 103 \\
4 & 10 & 4 & -7.4 & 103 \\
\hline \hline
\end{tabular}

term that varies with change in the magnetic field (e.g., Refs. 23 and 24) while others treat it as an instantaneous change in the solid temperature (e.g., Refs. 20 and 21). Because the AMR system that is modeled is not specific, the model used is not highly important. The 1D numerical model by Ref. 23 was chosen because it allows the regenerator material properties to be specified relatively easily and the computational requirements are not prohibitive. The model was verified experimentally against results for the AMR device described by Ref. 28 and good agreement between predicted and experimental results were achieved. ${ }^{23}$

The model used in this paper assumes that the fluid and solid temperature profiles are functions of only the $x$-direction (flow direction) and that there is a uniform fluid flow in the flow channels. The regenerator housing and ends $(x=0$, and $x=L)$ are assumed adiabatic. During the blow periods the fluid enters the regenerator with the prescribed temperature of the hot thermal reservoir $\left(T_{\mathrm{H}}\right)$ or the cold reservoir $\left(T_{\mathrm{C}}\right)$, and the system operates at steady state. AMR performance is determined by solving the coupled 1D partial differential equations in space and time describing the temperature in the regenerator and in the fluid. Established correlations are used to determine important regenerator parameters such as the heat transfer between solid and fluid phases and thermal axial conduction. The model is flexible with respect to operating conditions, geometry, and material and fluid properties. The equipment external to the regenerator bed (e.g., the pumps, drive motor, etc.) are not explicitly modeled; their effect on the bed is felt through an imposed time variation in the fluid mass flow rate and magnetic field. The magnetic field profile is the same for each simulation while the fluid flow rate is varied. The numerical model starts from an initial temperature distribution and takes implicit time steps forward in time until cyclical steady state has been achieved. Steady state is defined as when the dimensionless value of the absolute change in energy of the regenerator from cycle to cycle is less than a specified tolerance. The 1D model has been implemented in MATLAB and the code may be downloaded at the following web address: http://sel.me.wisc.edu/publications.shtml.

The regenerator geometry modeled in this work is a packed sphere regenerator with either $0.25 \mathrm{~mm}$ diameter spheres or $0.50 \mathrm{~mm}$ diameter spheres. The heat transfer between the regenerator and fluid is determined from the Nusselt number, which is determined from the correlation suggested by Ref. 29 for heat transfer in a packed sphere bed. The modeled regenerator has a length of $60 \mathrm{~mm}$ and a cross sectional area of $4 \mathrm{~cm}^{2}$. The heat transfer fluid is water with constant properties. The cycle parameters used as inputs to the AMR model are shown in Table IV. The dwell ratio is the fraction of the cycle when there is no fluid flow for a given regenerator bed.

\section{RESULTS AND DISCUSSION}

The model was run for regenerators with 0.25 and 0.50 $\mathrm{mm}$ sphere diameters (corresponding to approximately 0.1 and $0.2 \mathrm{~mm}$ hydraulic diameters) for a range of fluid flow rates for the set of materials listed in Table I. The predicted 
TABLE IV. Process parameters inputs for the numerical AMR model.

\begin{tabular}{lcc}
\hline \hline Parameter & Value & Unit \\
\hline Cold reservoir temp $\left(T_{\mathrm{C}}\right)$ & 285 & $\mathrm{~K}$ \\
Hot reservoir temp $\left(T_{\mathrm{H}}\right)$ & 300 & $\mathrm{~K}$ \\
Cycle period $(\tau)$ & 0.5 & $\mathrm{~s}$ \\
Dwell ratio & $1 / 3$ & $\mathrm{~mm}$ \\
Sphere diameter & $0.25,0.5$ & \\
Porosity $(\epsilon)$ & 0.36 & $\mathrm{~T}$ \\
Magnetic field & 2 & $\mathrm{~cm}^{3}$ \\
Regenerator volume & 24 & $\mathrm{~g}$ \\
MCM mass & 121 & \\
Fluid flow rate $\left(\dot{m}_{\mathrm{f}}\right)$ & varied & \\
\hline \hline
\end{tabular}

cooling power as a function of fluid flow rate is shown in Fig. 3. The fluid flow rates in this section are normalized by the volume of fluid to the total regenerator volume. They are intentionally not presented as a function of regenerator utilization to illustrate how the fluid flow rate varies with each material

$$
\overline{\dot{V}}=\frac{\dot{m}_{f} \rho_{f}}{A_{c} L}
$$

where $L$ is the length of the regenerator. The normalized flow rate indicates how many times the volume of the regenerator could be replaced by the fluid per second.

The results in Fig. 3 show some interesting trends. If $\Delta T_{\text {ad }}$ is held constant and the specific heat is increased (raising $\left.\Delta s_{\text {mag }}\right)$, the cooling capacity of the system increases for
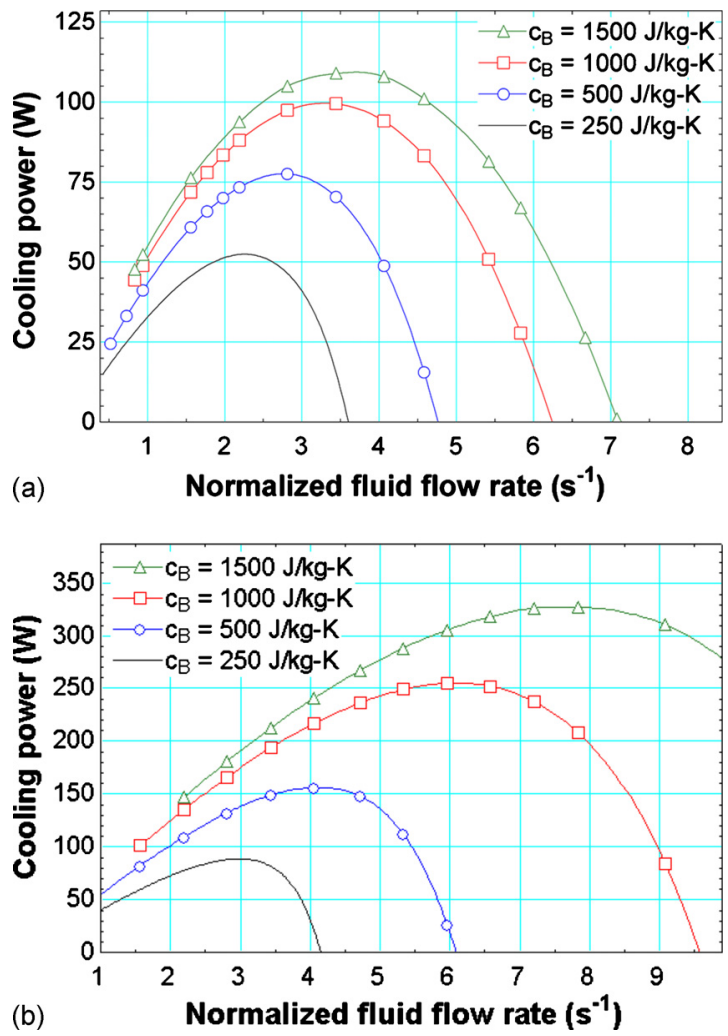

FIG. 3. (Color online) The refrigeration capacity as a function of normalized flow rate for materials with a constant $\Delta T_{\text {ad }}$ and varying specific heat for an AMR with (a) $0.5 \mathrm{~mm}$ and (b) $0.25 \mathrm{~mm}$ spheres.
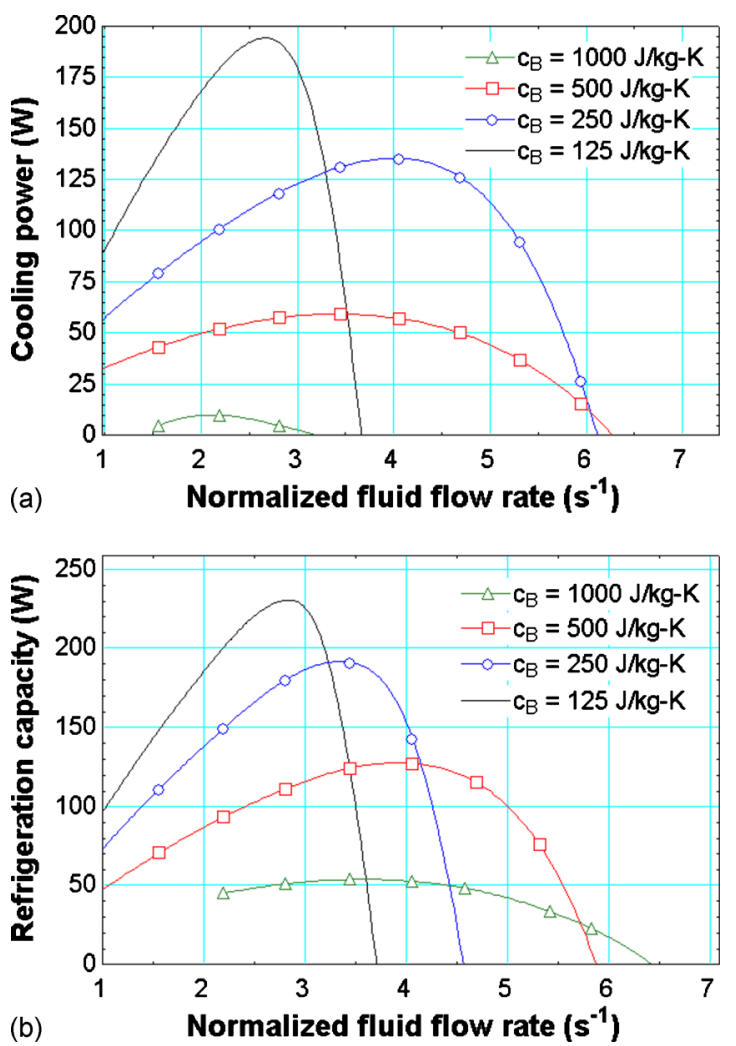

FIG. 4. (Color online) The refrigeration capacity as a function of normalized flow rate for materials with a constant $\Delta s_{\text {mag }}$ and varying specific heat for an AMR with (a) $0.5 \mathrm{~mm}$ and (b) $0.25 \mathrm{~mm}$ spheres.

all flow rates, but the flow rate must increase significantly to realize the full potential of the materials with higher thermal mass. The effect of increasing $\Delta s_{\text {mag }}$ while holding $\Delta T_{\text {ad }}$ constant is highly dependent on regenerator geometry. Figure 3 shows that the increase in cooling capacity with increasing specific heat is much greater for the regenerator with a smaller sphere size and higher heat transfer area. Because regenerators made from MCMs with high specific heat require high fluid flow rates, pumping losses will also increase and may become a significant loss mechanism.

The same procedure was performed on the set of material properties with constant $\Delta s_{\text {mag }}$ and varying specific heat and, therefore, varying $\Delta T_{\mathrm{ad}}$ and the results are shown in Fig. 4.

Figure 4 shows that AMR performance is significantly influenced by $\Delta T_{\text {ad }}$ of the material, even when $R_{\mathrm{CAP}}$ is held constant. As the specific heat increases, $\Delta T_{\text {ad }}$ and the refrigeration capacity tend toward zero. Materials with high $\Delta T_{\text {ad }}$ but low specific heat perform well at low fluid flow rates, but quickly become overwhelmed and are not suitable for high flow rate applications. Results for this set of materials also show that $\Delta T_{\text {ad }}$ is a more important property for regenerators with lower heat transfer performance, i.e., the regenerator with larger sphere diameter. AMR systems using materials with high $\Delta T_{\text {ad }}$ and low specific heat also have the advantage of being able to generate high cooling power at low fluid flow rates. This illustrates that heat transfer between the solid and fluid is an important phenomenon in AMR systems and 


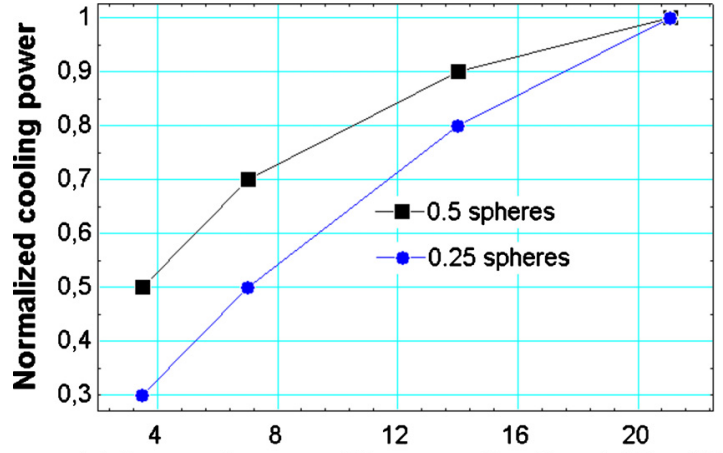

(a) Entropy change with magnetization ( $-\mathrm{J} / \mathbf{k g}-\mathrm{K}$ )

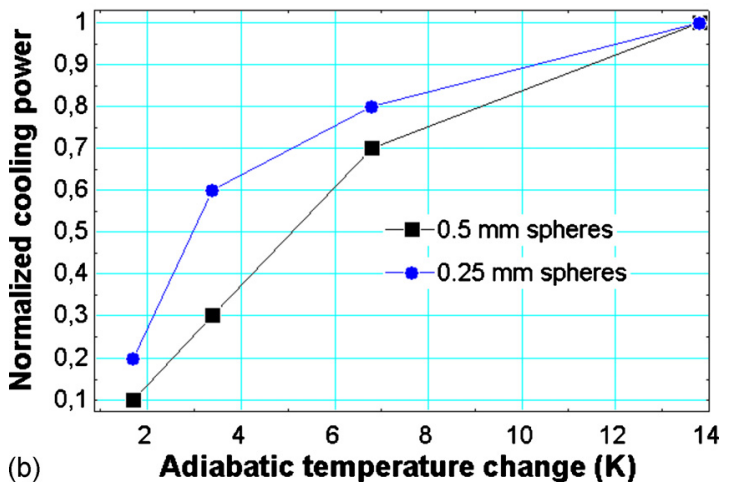

FIG. 5. (Color online) The normalized maximum refrigeration capacity as a function of (a) $\Delta s_{\text {mag }}$ and (b) $\Delta T_{\text {ad }}$ for an AMR with 0.5 and $0.25 \mathrm{~mm}$ spheres.

cannot be ignored when evaluating MCM properties. Figures 3 and 4 are summarized in Fig. 5 by normalizing the cooling capacity for each set of material.

The cooling power in Fig. 5 was normalized so that the maximum cooling power produced for each series is equal to one to study the relative impact of changes in either $\Delta T_{\text {ad }}$ or $\Delta s_{\text {mag }}$ on each system. The figure shows that the cooling power of regenerators with relatively low heat transfer performance is more dependent on $\Delta T_{\text {ad }}$ than $\Delta s_{\text {mag }}$, as a $50 \%$ reduction in $\Delta T_{\text {ad }}$ reduces cooling power by approximately $30 \%$ while a $67 \%$ reduction in $\Delta s_{\text {mag }}$ is needed for the same reduction in cooling power. The regenerator with smaller spheres is more sensitive to $\Delta s_{\mathrm{mag}}$, as it has the ability to transfer the magnetic work over a smaller temperature difference between the solid and fluid. However, both regenerators in Fig. 5 are highly dependent on $\Delta T_{\mathrm{ad}}$ of the regenerator material. Experiments on prototype AMR system have suggested that Gd regenerators often outperform similar devices that use materials with higher $\Delta s_{\text {mag }}$ but lower $\Delta T_{\text {ad. }}{ }^{4}$

Finally, the AMR performance for materials with constant $R_{\mathrm{CAP}}$ and varying shapes of the magnetocaloric effect was predicted, and the results are shown in Fig. 6. The figure shows that the material with the highest performance is that with a constant $\Delta s_{\text {mag. }}$. Cooling capacity can decrease by $50 \%$ for materials with the same $R_{\mathrm{CAP}}$ due to the shape of the specific heat and $\Delta s_{\text {mag }}$ curves. One explanation for this phenomenon is that the magnetocaloric effect is unevenly distributed when the material shows a pronounced peak in $\Delta s_{\text {mag }}$ with respect to temperature. Because the heat transfer and fluid flow are equal throughout the regenerator, portions

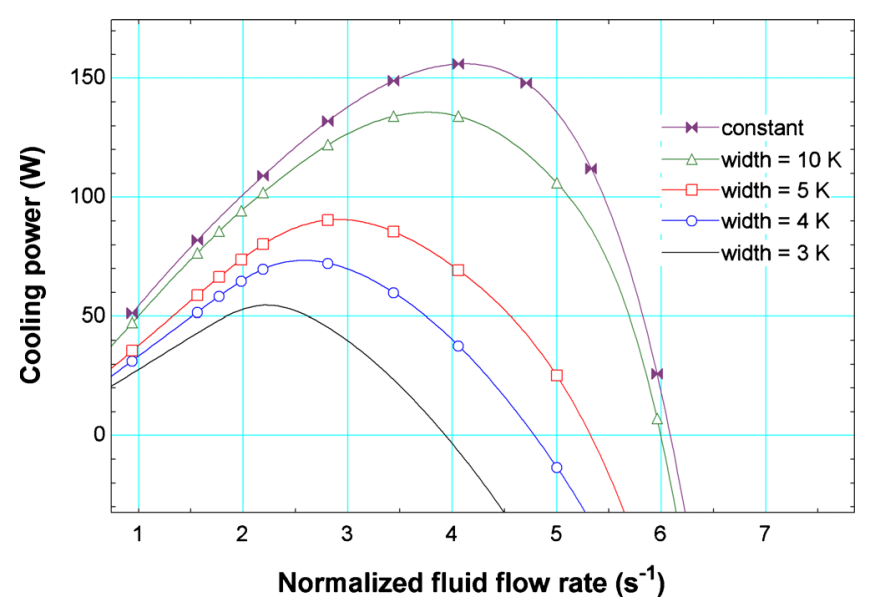

FIG. 6. (Color online) The cooling power as a function of normalized fluid flow rate for the material set listed in Table III for a regenerator with 0.25 $\mathrm{mm}$ spheres.

of the bed operating at temperatures further away from the Curie temperature underperform relative to other parts of the regenerator and the system performance decreases. Therefore, for a single material regenerator, MCMs with sharp peaks in the magnetocaloric effect are less attractive than those with an equal $R_{\mathrm{CAP}}$ and a more evenly distributed magnetocaloric effect.

The results presented here are for packed sphere regenerators but the general findings should also apply to parallel plate regenerators or other regenerator geometries. Studying the governing equations for the AMR system shows that the most important regenerator parameters are heat transfer performance and heat capacities of the solid and fluid. Therefore, any AMR system using similar materials and regenerators with similar heat transfer performance should exhibit the same trends. Pressure drop and axial conduction losses were not considered in this study, and those losses would not affect results presented in this paper if alternative regenerator geometries were modeled.

\section{CONCLUSIONS}

The effect of varying $\Delta s_{\text {mag }}$ and $\Delta T_{\text {ad }}$ were investigated using a $1 \mathrm{D}$ numerical AMR model. The results showed that the cooling power even for regenerators with high heat transfer performance (a regenerator of packed $0.25 \mathrm{~mm}$ spheres) is highly dependent on $\Delta T_{\text {ad }}$ of the regenerator material. This indicates that reporting $\Delta s_{\text {mag }}$ alone is not sufficient for characterizing MCMs and the common techniques to predict AMR performance based only on $\Delta s_{\text {mag }}$ data such as $R_{\text {CAP }}$ or RCP ignore the important heat transfer interaction between the solid and fluid in an AMR. The numerical simulations performed in this work also suggest that $\Delta T_{\text {ad }}$ is more important than $\Delta s_{\text {mag }}$ when the fluid flow rate is relatively low or the heat transfer effectiveness in the regenerator is low. Because materials with high $\Delta s_{\text {mag }}$ and high specific heat require larger fluid flow rates to realize their performance potential, pumping losses may become large and reduce system efficiency.

Finally, it was shown that materials with pronounced spikes in $\Delta s_{\text {mag }}$ show reduced performance compared to ma- 
terials with constant $\Delta s_{\text {mag }}$ and equal $R_{\mathrm{CAP}}$. This could make materials that exhibit a large peak in $\Delta s_{\text {mag }}$ over a narrow temperature band less desirable for AMR applications, especially for a single material regenerator. MCM research should emphasize developing materials with high $\Delta T_{\text {ad }}$ over a broad temperature range rather than solely increasing peak values of $\Delta s_{\text {mag }}$.

\section{ACKNOWLEDGMENTS}

The authors would like to acknowledge the support of the Programme Commission on Energy and Environment (EnMi) (Contract No. 2104-06-0032) which is part of the Danish Council for Strategic Research.

${ }^{1}$ K. A. Gschneidner, Jr., V. K. Pecharsky, and A. O. Tsokol, Rep. Prog. Phys. 68, 1479 (2005).

${ }^{2}$ W. F. Giauque and D. P. MacDougall, Phys. Rev. 43, 768 (1933).

${ }^{3}$ G. Brown, J. Appl. Phys. 47, 3673 (1976).

${ }^{4}$ B. Yu, M. Liu, P. W. Egolf, and A. Kitanovski, Int. J. Refrig. 33, 1029 (2010).

${ }^{5}$ K.A. Gschneidner, Jr. and V. Pecharsky, Int. J. Refrig. 31, 945 (2008).

${ }^{6}$ K. A. Gschneidner, Jr. and V. K. Pecharsky, Phys. Rev. Lett. 85, 4190 (2000).

${ }^{7}$ A. Fujita, S. Fujieda, Y. Hasegawa, and K. Fukamichi, Phys. Rev. B 67, 104416 (2003)

${ }^{8}$ E. Brück, M. Ilyn, A. M. Tishin, and O. Tegus, J. Magn. Magn. Mater. 290-291, 8 (2005).

${ }^{9}$ L. Mañosa, D. González-Alonso, A. Planes, E. Bonnot, M. Barrio, J.-L. Tamarit, S. Aksoy, and M. Acet, Nature Mater. 9, 478 (2010).

${ }^{10}$ J. Lyubina, R. Schäfer, N. Martin, L. Schultz, and O. Gutfleisch, Adv. Mater. (Weinheim, Ger.) 22, 3735 (2010).

${ }^{11}$ B. R. Hansen, L. T. Kuhn, C. R. H. Bahl, M. Lundberg, C. Ancona-Torres, and M. Katter, J. Magn. Magn. Mater. 322, 3447 (2010).

${ }^{12}$ S. A. Nikitin, K. P. Skokov, Y. S. Koshik'ko, Y. G. Pastushenkov, and T.
I. Ivanova, Phys. Rev. Lett. 105, 137205 (2010).

${ }^{13}$ R. Bjørk, C. Bahl, and M. Katter, J. Magn. Magn. Mater. 322, 3882 (2010).

${ }^{14}$ A. Rowe and A. Tura, Int. J. Refrig. 29, 1286 (2006).

${ }^{15} \mathrm{H}$. Hausen, Heat Transfer in Counterflow, Parallel-Flow and Cross-Flow (McGraw-Hill, New York, 1983).

${ }^{16}$ A. M. Tishin and Y. I. Spichkin, The Magnetocaloric Effect and its Applications (Institute of Physics, Temple Back, Bristol, UK, 2003).

${ }^{17}$ S. Dan'kov, A. Tishin, V. Pecharsky, and K. A. Gschneidner, Jr., Phys. Rev. B 57, 3478 (1998)

${ }^{18}$ A. Fujita, Y. Akamatsu, and K. Fukamichi, J. Appl. Phys. 85, 4756 (1999).

${ }^{19}$ S. Fujieda, Y. Hasegawa, A. Fujita, and K. Fukamichi, J. Appl. Phys. 95, 2429 (2004).

${ }^{20}$ M. Risser, C. Vasile, T. Engel, B. Keith, and C. Muller, Int. J. Refrig. 33, 973 (2010).

${ }^{21}$ G. Tagliafico, F. Scarpa, and F. Canepa, Int. J. Refrig. 33, 286 (2010).

${ }^{22} \mathrm{~S}$. Jacobs, in Proceedings of the Third International Conference Magnetic Refrigeration at Room Temperature, Des Moines, Iowa, USA, 2009, p. 267.

${ }^{23} \mathrm{~K}$. Engelbrecht, A numerical model of an active magnetic regenerator refrigerator with experimental validation, Ph.D. thesis, University of Wisconsin, 2008.

${ }^{24}$ K. Nielsen, C. Bahl, A. Smith, N. Pryds, and J. Hattel, Int. J. Refrig. 33, 753 (2010).

${ }^{25}$ P. A. Oliveira, P. Trevizoli, J. R. Barbosa, Jr., and A. Prata, Proceedings of the Third International Conference on Magnetic Refrigeration at Room Temperature, Des Moines, Iowa, USA, 2009, p. 283.

${ }^{26} \mathrm{M}$. Liu and B. F. Yu, in Proceedings of the Fourth International Conference on Magnetic Refrigeration at Room Temperature, Baotou, Inner Mongolia, China, 2010, p. 477.

${ }^{27}$ J. Bouchard, H. Nesreddine, and N. Galanis, Int. J. Refrig. 52, 1223 (2009).

${ }^{28}$ C. Zimm, A. Boeder, J. Chell, A. Sternberg, A. Fujita, S. Fujieda, and K. Fukamichi, Int. J. Refrig. 29, 1302 (2006)

${ }^{29}$ M. Kaviany, Principles of Heat Transfer in Porous Media (Springer, New York, 1995). 